\title{
SLC2A1 Gene
}

National Cancer Institute

\section{Source}

National Cancer Institute. SLC2A1 Gene. NCI Thesaurus. Code C28572.

This gene is involved in glucose transport. 